\title{
Elevated excitatory neurotransmitter levels in the fibromyalgia brain
}

\author{
Richard E Harris* \\ See related research by Fayed et al., http://arthritis-research.com/content/12/4/R134
}

\begin{abstract}
Consistent brain imaging findings demonstrate that neurobiological factors may contribute to the pathology of 'central' pain states such as fibromyalgia (FM). Studies using proton magnetic resonance spectroscopy suggest that glutamate (Glu), a key excitatory neurotransmitter, may be present in higher concentrations within the brains of FM patients. This neurotransmitter imbalance is present in multiple brain regions that have been implicated in processing pain information. However, it is unknown if elevated Glu is acting at the synapse. New investigations are needed to investigate the molecular action of Glu in FM and to investigate these findings during treatment that modulates glutamatergic neurotransmission.
\end{abstract}

The occurrence of central neurobiological factors in chronic pain states is becoming increasingly observed. For fibromyalgia (FM), this is certainly true as evidenced by the recent article by Fayed and colleagues in the previous issue of Arthritis Research and Therapy [1]. These authors used a brain imaging tool called proton magnetic resonance spectroscopy ( $\left.{ }^{1} \mathrm{H}-\mathrm{MRS}\right)$, which can assess the concentration of specific metabolites in the human brain, and looked for altered levels of brain neurotransmitters in individuals with FM. Of particular importance to the field of neurobiology, ${ }^{1} \mathrm{H}-\mathrm{MRS}$ methods allow for the detection of the brain's major excitatory and inhibitory neurotransmitters, glutamate (Glu) and gamma amino-butyric acid (GABA), respectively. To date, this method has been used frequently in disorders of the brain, such as depression [2], Alzheimer's disease [3], and epilepsy [4]; recently, however, it is

*Correspondence: reharris@med.umich.edu

Department of Anesthesiology, Chronic Pain and Fatigue Research Center,

University of Michigan, 24 Frank Lloyd Wright Dr., P.O. Box 385 Lobby M, Ann Arbor, MI 48106, USA becoming increasingly evident that chronic 'central' pain states such as FM may also have altered brain neurotransmitter levels, as evidenced by new ${ }^{1} \mathrm{H}$-MRS studies [5-7].

Fayed and colleagues performed a cross-sectional ${ }^{1} \mathrm{H}$ MRS investigation of ten patients with FM and ten sexand age-matched healthy controls, looking at four specific brain regions that are thought to be somewhat involved in pain processing: the sensorimotor cortex, the thalamus, the hippocampus, and the posterior gyrus (posterior cingulate). The authors report elevated levels of Glx, a combined measure of Glu and glutamine, within the posterior cingulate of FM patients. Moreover, they observed negative correlations between Glx in the patient group and pressure pain thresholds, and positive associations with these metabolites and scores on the Fibromyalgia Impact Questionnaire, suggesting that elevated levels of Glx in the posterior cingulate are associated with increased pain and tenderness in FM. The posterior cingulate region of the brain is also a key component of the default mode network (DMN), a constellation of brain regions activated during self-referential thinking. In FM patients, the DMN appears to have greater functional connections with the insula, a key pain processing region, and this enhanced connectivity is directly related to the spontaneous clinical pain in these patients [8]. In aggregate, these data point towards a potential molecular basis for some of the functional neuroimaging results observed in FM $[9,10]$ : namely, an elevation in brain excitatory neurotransmitters that could result in neuronal hyperexcitability. This is not the first such study of ${ }^{1} \mathrm{H}$ MRS in FM. Harris and colleagues [5] demonstrated elevated levels of Glu and Glx within the posterior insula of FM patients, and Valdés and colleagues [6] recently reported that Glx was elevated in the amygdala, an emotional and pain processing brain region. These findings, reported by three separate groups, are beginning to paint a picture of a more global increase in the concentration of the excitatory neurotransmitters in the FM brain. It is important to note that across these three studies, elevated levels of Glu and Glx were not observed in all regions studied. Fayed and colleagues 
found elevated Glx in the posterior cingulate but not the thalamus or the somatosensory cortex. Valdés and colleagues found elevated Glx in the amygdala but not the thalamus or prefrontal cortex, and Harris and colleagues found elevated Glx in the posterior but not the anterior insula. Therefore, although multiple brain regions may have elevated Glu in FM, this does not appear to be the case for every region examined.

As mentioned above, it is tempting to conclude that the reason why previous functional magnetic resonance imaging studies of FM have observed augmented neural activity is due to an elevation of Glu leading to neuronal hyperexcitability. At the present moment, however, we cannot conclude this for the following reasons: first, ${ }^{1} \mathrm{H}$ MRS-derived levels of Glu are often contaminated in part by glutamine [11], thus precluding our ability to assign the elevated levels solely to Glu at this time; second, within brain tissue, Glu exists in the neurotransmitter pool as well as a metabolic pool [12] and we do not know if the neurotransmitter pool is where the elevation is occurring; and finally, the cellular compartment from where the elevated Glu signal is originating is unknown. The ${ }^{1} \mathrm{H}$-MRS signal arises from gray and white matter and is an ensemble average of multiple different cell types, including astrocytes, glia, and neurons. Therefore, the elevated Glu signal might be at regions distant from the synapse.

Unfortunately, answering these questions will require novel preclinical research, as they cannot be directly assessed with the existing technologies in humans. That said, the work by Fayed and colleagues and others contributes significantly to the field. It places a key neurotransmitter to the forefront of our thinking behind the molecular processes involved in FM. It also opens the door for ${ }^{1} \mathrm{H}$-MRS studies of other pain states that may also be mediated by central factors. Finally, these data also suggest that pharmacologic interventions that specifically target glutamatergic neurotransmission may be effective in these complex patients. Research in these questions is greatly needed.

Abbreviations

DMN, default mode network; FM, fibromyalgia; Glu, glutamate; GIx, combined measure of Glu and glutamine; ${ }^{1} \mathrm{H}-\mathrm{MRS}$, proton magnetic resonance

spectroscopy.
Competing interests

REH has received funding for salary support and consulting from Pfizer Inc.

Published: 1 October 2010

\section{References}

1. Fayed N, Garcia-Campayo J, Magallón R, Andrés-Bergareche H, Luciano JV, Andres E, Beltrán J: Localized 'H-NMR spectroscopy in patients with fibromyalgia: a controlled study of changes in cerebral glutamate/ glutamine, inositol, choline, and $\mathrm{N}$-acetylaspartate. Arthritis Res Ther 2010, 12:R134.

2. Sanacora G, Gueorguieva R, Epperson CN, Wu YT, Appel M, Rothman DL, Krystal JH, Mason GF: Subtype-specific alterations of gamma-aminobutyric acid and glutamate in patients with major depression. Arch Gen Psychiatry 2004, 61:705-713.

3. Jessen F, Gür O, Block W, Ende G, Frölich L, Hammen T, Wiltfang J, Kucinski T, Jahn H, Heun R, Maier W, Kölsch H, Kornhuber J, Träber F: A multicenter (1) H-MRS study of the medial temporal lobe in AD and MCl. Neurology 2009, 72:1735-1740.

4. Helms G, Ciumas C, Kyaga S, Savic I: Increased thalamus levels of glutamate and glutamine (G|x) in patients with idiopathic generalised epilepsy. J Neurol Neurosurg Psychiatry 2006, 77:489-494.

5. Harris RE, Sundgren PC, Craig AD, Kirshenbaum E, Sen A, Napadow V, Clauw DJ: Elevated insular glutamate (Glu) in fibromyalgia (FM) is associated with experimental pain. Arthritis Rheum 2009, 60:3146-3152.

6. Valdés M, Collado A, Bargalló N, Vázquez M, Rami L, Gómez E, Salamero M: Increased glutamate/glutamine compounds in the brains of patients with fibromyalgia: a magnetic resonance spectroscopy study. Arthritis Rheum 2010, 62:1829-1836.

7. Harris RE, Sundgren PC, Pang Y, Hsu M, Petrou M, Kim SH, McLean SA, Gracely RH, Clauw DJ: Dynamic levels of glutamate within the insula are associated with improvements in multiple pain domains in fibromyalgia. Arthritis Rheum 2008, 58:903-907.

8. Napadow V, LaCount L, Park K, As-Sanie S, Clauw D, Harris RE: Intrinsic brain connectivity in fibromyalgia is associated with chronic pain intensity. Arthritis Rheum 2010, 62:2545-2555.

9. Gracely RH, Petzke F, Wolf JM, Clauw DJ: Functional magnetic resonance imaging evidence of augmented pain processing in fibromyalgia. Arthritis Rheum 2002, 46:1333-1343.

10. Cook DB, Lange G, Ciccone DS, Liu WC, Steffener J, Natelson BH: Functional imaging of pain in patients with primary fibromyalgia. $J$ Rheumatol 2004, 31:364-378.

11. de Graaf RA: In Vivo NMR Spectroscopy: Principles and Techniques. New York: John Wiley \& Sons; 1998.

12. Nedergaard M, Takano T, Hansen AJ: Beyond the role of glutamate as a neurotransmitter. Nat Rev Neurosci 2002, 3:748-755.

doi:10.1186/ar3136

Cite this article as: Harris RE: Elevated excitatory neurotransmitter levels in the fibromyalgia brain. Arthritis Research \& Therapy 2010, 12:141. 